\title{
Logarithmic $O\left(\alpha_{s}^{3}\right)$ contributions to the DIS Heavy Flavor Wilson Coefficients at $Q^{2} \gg m^{2}$
}

\section{Isabella Bierenbaum*}

Instituto de Física Corpuscular, CSIC-Universitat de València, Apartado de Correos 22085,

E-46071 Valencia, Spain

E-mail: Isabella.Bierenbaumeific.uv.es

\section{Johannes Blümlein}

Deutsches Elektronen-Synchrotron, DESY, Platanenallee 6, D-15638 Zeuthen, Germany

E-mail: Johannes.Bluemlein@desy.de

\section{Sebastian Klein ${ }^{\S}$}

Institut für theoretische Teilchenphysik und Kosmologie, RWTH Aachen University, D-52056

Aachen, Germany

E-mail: sklein@physik.rwth-aachen.de

The logarithmic contributions to the massive twist-2 operator matrix elements for deep-inelastic scattering are calculated to $O\left(\alpha_{s}^{3}\right)$ for general values of the Mellin variable $N$.

XVIII International Workshop on Deep-Inelastic Scattering and Related Subjects, DIS 2010 April 19-23, 2010

Firenze, Italy

\footnotetext{
* Supported in part by Generalitat Valenciana Grant No. PROMETEO/2008/69.

$\dagger$ Speaker.

¥Supported in part by SFB-TR/9 and EU TMR network HEPTOOLS.

${ }^{\S}$ Supported in part by SFB-TR/9.
} 


\section{Introduction}

The heavy flavor contributions to deep-inelastic scattering (DIS) are rather large in the small $x$ region. With the current DIS data a precision of $\sim 1 \%$ is reached [1] for $F_{2}\left(x, Q^{2}\right)$. This requires to describe the heavy flavor corrections to 3-loop order, to perform a consistent next-to-next-to leading order (NNLO) analysis, to measure the strong coupling constant $\alpha_{s}\left(M_{Z}^{2}\right)$ and the unpolarized twist-2 parton distribution functions at highest precision possible, cf. [2]]. In the region $Q^{2} / \mathrm{m}^{2} \geq 10$ one may compute all contributions except the power suppressed terms, $\propto\left(m^{2} / Q^{2}\right)^{k}, k \geq 1$ using the factorization theorem given in Ref. [3]. Here, the massive Wilson coefficients factorize into massive operator matrix elements (OMEs), $A_{i j}$, and the massless Wilson coefficients, which are known to $O\left(\alpha_{s}^{3}\right)$ 㺻. The $O\left(\alpha_{s}^{3}\right)$ Mellin moments of the massive operator matrix elements up to $N=10 \ldots 14$, depending on the process, were computed in [5]. The calculation was performed relating the moments of the massive operator matrix elements to massive tadpoles and using MATAD [6]. In Ref. [5] also the complete renormalization for a single massive quark has been derived. Different other contributions, needed in the renormalization process, were computed at general values of $N$ in Refs. [7]. For the structure function $F_{L}\left(x, Q^{2}\right)$ the asymptotic corrections to $O\left(\alpha_{s}^{3}\right)$ are known for general values of $N$ [8]. They are, however, only applicable at scales $Q^{2} / m^{2} \geq 800$. For transversity the matrix elements were computed for general $N$ at 2-loop order and a series of moments at 3-loop order in [9]. Very recently, the general $N$ results at $O\left(\alpha_{s}^{3}\right)$ for $F_{2}\left(x, Q^{2}\right)$ for the color coefficients $\propto n_{f}$ have been completed [10, 11]. These computations use modern summation technologies encoded in the package Sigma [12] and the results can be expressed in terms of nested harmonic sums [13]. From the single pole terms in the massive computations of Refs. [5, 9, 10, 11] the corresponding contributions to the 3-loop anomalous dimensions were derived, either for the respective moments [14], or at general values of $N$ [16].

In this note we report on the logarithmic $O\left(\alpha_{s}^{3}\right)$ contributions to the massive operator matrix elements, cf. also [17]. They are known for general values of $N$ and depend on the 3-loop anomalous dimensions and massive OMEs up to $O\left(\alpha_{s}^{2}\right)$.

\section{The heavy flavor Wilson coefficients in the asymtotic region}

The heavy flavor correction to the structure function $F_{2}\left(x, Q^{2}\right)$ with $n_{f}$ massless and one heavy flavor reads, [5] :

$$
\begin{aligned}
& F_{(2, L)}^{Q \bar{Q}}\left(x, n_{f}, Q^{2}, m^{2}\right)=\sum_{k=1}^{n_{f}} e_{k}^{2}\left\{L_{q,(2, L)}^{\mathrm{NS}}\left(x, n_{f}+1, \frac{Q^{2}}{m^{2}}, \frac{m^{2}}{\mu^{2}}\right) \otimes\left[f_{k}\left(x, \mu^{2}, n_{f}\right)+f_{\bar{k}}\left(x, \mu^{2}, n_{f}\right)\right]\right. \\
& \left.+\frac{1}{n_{f}}\left[L_{q,(2, L)}^{\mathrm{PS}}\left(x, n_{f}+1, \frac{Q^{2}}{m^{2}}, \frac{m^{2}}{\mu^{2}}\right) \otimes \Sigma\left(x, \mu^{2}, n_{f}\right)+L_{g,(2, L)}^{\mathrm{S}}\left(x, n_{f}+1, \frac{Q^{2}}{m^{2}}, \frac{m^{2}}{\mu^{2}}\right) \otimes G\left(x, \mu^{2}, n_{f}\right)\right]\right\} \\
& +e_{Q}^{2}\left[H_{q,(2, L)}^{\mathrm{PS}}\left(x, n_{f}+1, \frac{Q^{2}}{m^{2}}, \frac{m^{2}}{\mu^{2}}\right) \otimes \Sigma\left(x, \mu^{2}, n_{f}\right)+H_{g,(2, L)}^{\mathrm{S}}\left(x, n_{f}+1, \frac{Q^{2}}{m^{2}}, \frac{m^{2}}{\mu^{2}}\right) \otimes G\left(x, \mu^{2}, n_{f}\right)\right],
\end{aligned}
$$

with boundaries for the Mellin integral $\left[x\left(1+4 m^{2} / Q^{2}\right), 1\right]$, and $e_{i}$ the quark charges. Here the different Wilson coefficients are denoted by $L_{i}, H_{i}$ in case the photon couples to a light (L) or the 
heavy $(\mathrm{H})$ quark. For $Q^{2} \gg m^{2}$ they can be expressed in terms of the massive OMEs $A_{i j}$ and the massless Wilson coefficients $C_{j}$. To $O\left(a_{s}^{3}\right)$ they read $\left(a_{s}=\alpha_{s} /(4 \pi)\right)$

$$
\begin{aligned}
L_{q,(2, L)}^{\mathrm{NS}}\left(n_{f}+1\right)= & a_{s}^{2}\left[A_{q q, Q}^{(2), \mathrm{NS}}\left(n_{f}+1\right) \delta_{2}+\hat{C}_{q,(2, L)}^{(2), \mathrm{NS}}\left(n_{f}\right)\right] \\
+ & a_{s}^{3}\left[A_{q q, Q}^{(3), \mathrm{NS}}\left(n_{f}+1\right) \delta_{2}+A_{q q, Q}^{(2), \mathrm{NS}}\left(n_{f}+1\right) C_{q,(2, L)}^{(1), \mathrm{NS}}\left(n_{f}+1\right)+\hat{C}_{q,(2, L)}^{(3), \mathrm{NS}}\left(n_{f}\right)\right] \\
L_{q,(2, L)}^{\mathrm{PS}}\left(n_{f}+1\right)= & a_{s}^{3}\left[A_{q q, Q}^{(3), \mathrm{PS}}\left(n_{f}+1\right) \delta_{2}+A_{g q, Q}^{(2)}\left(n_{f}\right) n_{f} \tilde{C}_{g,(2, L)}^{(1)}\left(n_{f}+1\right)+n_{f} \hat{\tilde{C}}_{q,(2, L)}^{(3), \mathrm{PS}}\left(n_{f}\right)\right] \\
L_{g,(2, L)}^{\mathrm{S}}\left(n_{f}+1\right)= & a_{s}^{2} A_{g g, Q}^{(1)}\left(n_{f}+1\right) n_{f} \tilde{C}_{g,(2, L)}^{(1)}\left(n_{f}+1\right)+a_{s}^{3}\left[A_{q g, Q}^{(3)}\left(n_{f}+1\right) \delta_{2}\right. \\
& +A_{g g, Q}^{(1)}\left(n_{f}+1\right) n_{f} \tilde{C}_{g,(2, L)}^{(2)}\left(n_{f}+1\right)+A_{g g, Q}^{(2)}\left(n_{f}+1\right) n_{f} \tilde{C}_{g,(2, L)}^{(1)}\left(n_{f}+1\right) \\
& \left.+A_{Q g}^{(1)}\left(n_{f}+1\right) n_{f} \tilde{C}_{q,(2, L)}^{(2), \mathrm{PS}}\left(n_{f}+1\right)+n_{f} \tilde{\widetilde{C}}_{g,(2, L)}^{(3)}\left(n_{f}\right)\right] \\
H_{q,(2, L)}^{\mathrm{PS}}\left(n_{f}+1\right)= & a_{s}^{2}\left[A_{Q q}^{(2), \mathrm{PS}}\left(n_{f}+1\right) \delta_{2}+\tilde{C}_{q,(2, L)}^{(2), \mathrm{PS}}\left(n_{f}+1\right)\right]+a_{s}^{3}\left[A_{Q q}^{(3), \mathrm{PS}}\left(n_{f}+1\right) \delta_{2}\right. \\
& +\tilde{C}_{q,(2, L)}^{(3) \mathrm{PS}}\left(n_{f}+1\right)+A_{g q, Q}^{(2)}\left(n_{f}+1\right) \tilde{C}_{g,(2, L)}^{(1)}\left(n_{f}+1\right) \\
& \left.+A_{Q q}^{(2), \mathrm{PS}}\left(n_{f}+1\right) C_{q,(2, L)}^{(1), \mathrm{NS}}\left(n_{f}+1\right)\right] \\
H_{g,(2, L)}^{\mathrm{S}}\left(n_{f}+1\right)= & a_{s}\left[A_{Q g}^{(1)}\left(n_{f}+1\right) \delta_{2}+\tilde{C}_{g,(2, L)}^{(1)}\left(n_{f}+1\right)\right]+a_{s}^{2}\left[A_{Q g}^{(2)}\left(n_{f}+1\right) \delta_{2}\right. \\
& +A_{Q g}^{(1)}\left(n_{f}+1\right) C_{q,(2, L)}^{(1), \mathrm{NS}}\left(n_{f}+1\right)+A_{g g, Q}^{(1)}\left(n_{f}+1\right) \tilde{C}_{g,(2, L)}^{(1)}\left(n_{f}+1\right) \\
& \left.+\tilde{C}_{g,(2, L)}^{(2)}\left(n_{f}+1\right)\right]+a_{s}^{3}\left[A_{Q g}^{(3)}\left(n_{f}+1\right) \delta_{2}+A_{Q g}^{(2)}\left(n_{f}+1\right) C_{q,(2, L)}^{(1), \mathrm{NS}}\left(n_{f}+1\right)\right. \\
& +A_{g g, Q}^{(2)}\left(n_{f}+1\right) \tilde{C}_{g,(2, L)}^{(1)}\left(n_{f}+1\right)+A_{Q g}^{(1)}\left(n_{f}+1\right)\left\{C_{q,(2, L)}^{(2), \mathrm{NS}}\left(n_{f}+1\right)\right. \\
& \left.\left.+\tilde{C}_{q,(2, L)}^{(2) \mathrm{PS}}\left(n_{f}+1\right)\right\}+A_{g g, Q}^{(1)}\left(n_{f}+1\right) \tilde{C}_{g,(2, L)}^{(2)}\left(n_{f}+1\right)+\tilde{C}_{g,(2, L)}^{(3)}\left(n_{f}+1\right)\right]
\end{aligned}
$$

with $\delta_{2}=0(1)$ for $F_{L}\left(F_{2}\right)$ and $\hat{f}\left(n_{f}\right)=f\left(n_{f}+1\right)-f\left(n_{f}\right), \tilde{f}\left(n_{f}\right)=f\left(n_{f}\right) / n_{f}$. The massive OMEs depend on the ratio $m^{2} / \mu^{2}$, while the scale ratio of the massless Wilson coefficients is $\mu^{2} / Q^{2}$. The latter are pure functions of the momentum fraction $z$, or the Mellin variable $N$, if one sets $\mu^{2}=Q^{2}$. The massive OMEs obey then the general structure

$$
A_{i j}^{(3)}\left(\frac{m^{2}}{Q^{2}}\right)=a_{i j}^{(3), 3} \ln ^{3}\left(\frac{m^{2}}{Q^{2}}\right)+a_{i j}^{(3), 2} \ln ^{2}\left(\frac{m^{2}}{Q^{2}}\right)+a_{i j}^{(3), 1} \ln \left(\frac{m^{2}}{Q^{2}}\right)+a_{i j}^{(3), 0}
$$

\section{The matrix element $A_{Q g}^{(3)}(N)$}

In the following we present, as an example, the logarithmic expansion coefficients of Eq. (2.3), $a^{(3), k}, k \geq 1$, for the massive $\mathrm{OME} A_{Q g}^{(3)}(N)$ in the $\overline{\mathrm{MS}}$-scheme. They are given by :

$$
\begin{aligned}
& a_{Q g}^{(3), 3}=\frac{8\left(N^{2}+N+2\right) T_{F}}{9 N(N+1)(N+2)}\left[T _ { F } n _ { f } \left(C_{F}\left(\frac{P_{1}}{(N-1) N^{2}(N+1)^{2}(N+2)}-4 S_{1}\right)\right.\right. \\
& \left.\quad+C_{A}\left(4 S_{1}-\frac{8\left(N^{2}+N+1\right)}{(N-1) N(N+1)(N+2)}\right)\right)-8 T_{F}^{2}+C_{A}^{2}\left(-\frac{\left(11 N^{4}+22 N^{3}-59 N^{2}-70 N-48\right) S_{1}}{(N-1) N(N+1)(N+2)}\right.
\end{aligned}
$$




$$
\begin{aligned}
& \left.-12 S_{1}^{2}+\frac{2\left(N^{2}+N+1\right)\left(11 N^{4}+22 N^{3}-35 N^{2}-46 N-24\right)}{(N-1)^{2} N^{2}(N+1)^{2}(N+2)^{2}}\right)+C_{A} T_{F}\left(-\frac{56\left(N^{2}+N+1\right)}{(N-1) N(N+1)(N+2)}\right. \\
& \left.+28 S_{1}\right)+C_{F}^{2}\left(-3 \frac{\left(3 N^{2}+3 N+2\right)^{2}}{4 N^{2}(N+1)^{2}}+\frac{6 S_{1}\left(3 N^{2}+3 N+2\right)}{N(N+1)}-12 S_{1}^{2}\right)+C_{F} T_{F}\left(-16 S_{1}\right. \\
& \left.+\frac{2 P_{2}}{(N-1) N^{2}(N+1)^{2}(N+2)}\right)+C_{A} C_{F}\left(24 S_{1}^{2}-\frac{\left(N^{2}+N+6\right)\left(7 N^{2}+7 N+4\right) S_{1}}{(N-1) N(N+1)(N+2)}\right. \\
& \left.\left.-\frac{\left(3 N^{2}+3 N+2\right)\left(11 N^{4}+22 N^{3}-59 N^{2}-70 N-48\right)}{4(N-1) N^{2}(N+1)^{2}(N+2)}\right)\right] \text {. } \\
& a_{Q g}^{(3), 2}=4 T_{F}^{2} n_{f}\left(C _ { F } \left(-\frac{4\left(N^{2}+N+2\right)}{3 N(N+1)(N+2)}\left(S_{2}+S_{1}^{2}\right)+\frac{8\left(5 N^{3}+8 N^{2}+19 N+6\right) S_{1}}{9 N^{2}(N+1)(N+2)}\right.\right. \\
& \left.-\frac{P_{3}}{9(N-1) N^{4}(N+1)^{4}(N+2)^{3}}\right)+C_{A}\left(-\frac{8\left(5 N^{4}+20 N^{3}+47 N^{2}+58 N+20\right) S_{1}}{9 N(N+1)^{2}(N+2)^{2}}\right. \\
& \left.\left.+\frac{4\left(N^{2}+N+2\right)}{3 N(N+1)(N+2)}\left(2 S_{-2}+S_{2}+S_{1}^{2}\right)-\frac{2 P_{4}}{9(N-1) N^{2}(N+1)^{3}(N+2)^{3}}\right)\right)+2 C_{A}^{2} T_{F}( \\
& \frac{8\left(N^{2}+N+2\right)}{N(N+1)(N+2)}\left(2 S_{-2,1}-S_{1}^{3}-3 S_{2} S_{1}-S_{-3}-4 S_{-2} S_{1}-S_{3}\right)-\frac{2 P_{5} S_{1}^{2}}{3(N-1) N^{2}(N+1)^{2}(N+2)^{2}} \\
& +\frac{4 P_{6} S_{1}}{9(N-1)^{2} N^{3}(N+1)^{3}(N+2)^{3}}-\frac{4\left(N^{2}+N+2\right)\left(11 N^{4}+22 N^{3}-59 N^{2}-70 N-48\right) S_{-2}}{3(N-1) N^{2}(N+1)^{2}(N+2)^{2}} \\
& \left.+\frac{8 P_{7}}{9(N-1)^{2} N^{4}(N+1)^{4}(N+2)^{4}}-\frac{2\left(N^{2}+N+2\right)\left(11 N^{4}+22 N^{3}-83 N^{2}-94 N-72\right) S_{2}}{3(N-1) N^{2}(N+1)^{2}(N+2)^{2}}\right) \\
& +4 C_{A} T_{F}^{2}\left(\frac{4\left(N^{2}+N+2\right)}{N(N+1)(N+2)}\left(S_{1}^{2}+S_{2}+2 S_{-2}\right)+\frac{8\left(5 N^{4}+20 N^{3}-N^{2}-14 N+20\right) S_{1}}{9 N(N+1)^{2}(N+2)^{2}}\right. \\
& \left.-\frac{2 P_{8}}{9(N-1) N^{3}(N+1)^{3}(N+2)^{3}}\right)+2 C_{F}^{2} T_{F}\left(\frac{8\left(N^{2}+N+2\right)}{N(N+1)(N+2)}\left(3 S_{2} S_{1}-S_{1}^{3}+4 S_{-2} S_{1}+2 S_{3}-4 S_{-2,1}\right)\right. \\
& -\frac{16\left(N^{2}+N+2\right) S_{-2}}{N^{2}(N+1)^{2}(N+2)}-\frac{6\left(N^{2}+N+2\right)\left(3 N^{2}+3 N+2\right) S_{2}}{N^{2}(N+1)^{2}(N+2)}+\frac{2\left(3 N^{4}+14 N^{3}+43 N^{2}+48 N+20\right) S_{1}^{2}}{N^{2}(N+1)^{2}(N+2)} \\
& \left.-\frac{4 P_{9} S_{1}}{N^{3}(N+1)^{3}(N+2)}+\frac{P_{10}}{2 N^{4}(N+1)^{4}(N+2)}+\frac{16\left(N^{2}+N+2\right) S_{-3}}{N(N+1)(N+2)}\right)+4 C_{F} T_{F}^{2}( \\
& \left.\frac{4\left(N^{2}+N+2\right)}{3 N(N+1)(N+2)}\left(S_{2}-3 S_{1}^{2}\right)+\frac{8\left(5 N^{3}+14 N^{2}+37 N+18\right) S_{1}}{9 N^{2}(N+1)(N+2)}-\frac{P_{11}}{9(N-1) N^{4}(N+1)^{4}(N+2)^{3}}\right) \\
& +2 C_{F} C_{A} T_{F}\left(\frac{4\left(N^{2}+N+2\right)}{N(N+1)(N+2)}\left(4 S_{1}^{3}-2 S_{3}+4 S_{-2,1}-2 S_{-3}-3 S_{-2}\right)\right. \\
& +\frac{4 P_{12} S_{1}^{2}}{3(N-1) N^{2}(N+1)^{2}(N+2)^{2}}-\frac{4 P_{13} S_{1}}{9(N-1) N^{3}(N+1)^{3}(N+2)^{3}} \\
& \left.+\frac{P_{14}}{18(N-1) N^{3}(N+1)^{3}(N+2)^{3}}+\frac{4\left(N^{2}+N+2\right)\left(N^{4}+2 N^{3}+8 N^{2}+7 N+18\right) S_{2}}{3(N-1) N^{2}(N+1)^{2}(N+2)^{2}}\right) .
\end{aligned}
$$

$$
a_{Q g}^{(3), 1}=\frac{1}{2} \hat{\gamma}_{q g}^{(2)}\left(n_{f}\right)-\frac{n_{f}}{2} \hat{\gamma}_{q g}^{(2)}\left(n_{f}\right)+4 T_{F}^{2} n_{f}\left(C _ { F } \left(\frac{4\left(N^{2}+N+2\right)}{9 N(N+1)(N+2)}\left(4 S_{3}-S_{1}^{3}-3 S_{2} S_{1}\right)+\frac{4(3 N+2) S_{1}^{2}}{3 N^{2}(N+2)}\right.\right.
$$




$$
\begin{aligned}
& \left.+\frac{4\left(N^{4}-N^{3}-20 N^{2}-10 N-4\right) S_{1}}{3 N^{2}(N+1)^{2}(N+2)}+\frac{2 P_{15}}{3(N-1) N^{5}(N+1)^{5}(N+2)^{4}}+\frac{4 P_{16} S_{2}}{3(N-1) N^{3}(N+1)^{3}(N+2)^{2}}\right) \\
& +C_{A}\left(\frac{4\left(N^{2}+N+2\right)}{9 N(N+1)(N+2)}\left(S_{1}^{3}+9 S_{2} S_{1}+6 S_{-3}+12 S_{-2} S_{1}+8 S_{3}-12 S_{-2,1}\right)-\frac{4 P_{17} S_{1}}{3 N(N+1)^{3}(N+2)^{3}}\right. \\
& -\frac{4\left(N^{3}+8 N^{2}+11 N+2\right) S_{1}^{2}}{3 N(N+1)^{2}(N+2)^{2}}+\frac{4 P_{18}}{3(N-1) N^{4}(N+1)^{4}(N+2)^{4}}-\frac{4 P_{19} S_{2}}{3(N-1) N^{2}(N+1)^{2}(N+2)^{2}} \\
& \left.\left.+\frac{16\left(N^{2}-N-4\right) S_{-2}}{3(N+1)^{2}(N+2)^{2}}\right)\right)+2 C_{A}^{2} T_{F}\left(\frac { 8 ( N ^ { 2 } + N + 2 ) } { 3 N ( N + 1 ) ( N + 2 ) } \left(12 S_{-2,1} S_{1}-S_{1}^{4}-9 S_{2} S_{1}^{2}-8 S_{3} S_{1}-6 S_{-3} S_{1}\right.\right. \\
& \left.-12 S_{-2} S_{1}^{2}\right)-\frac{2 P_{20} S_{1}^{3}}{9(N-1) N^{2}(N+1)^{2}(N+2)^{2}}+\frac{2 P_{21} S_{1}^{2}}{3(N-1) N^{2}(N+1)^{3}(N+2)^{3}} \\
& -\frac{2 P_{22} S_{1}}{3(N-1) N^{4}(N+1)^{4}(N+2)^{4}}-\frac{2 P_{23} S_{2} S_{1}}{(N-1) N^{2}(N+1)^{2}(N+2)^{2}}-\frac{2 P_{24}}{3(N-1)^{2} N^{5}(N+1)^{5}(N+2)^{5}} \\
& +\frac{4\left(N^{2}+N+2\right)\left(11 N^{4}+22 N^{3}-35 N^{2}-46 N-24\right)}{9(N-1) N^{2}(N+1)^{2}(N+2)^{2}}\left(6 S_{-2,1}-4 S_{3}-3 S_{-3}\right) \\
& -\frac{8\left(N^{2}-N-4\right)\left(11 N^{4}+22 N^{3}-35 N^{2}-46 N-24\right) S_{-2}}{3(N-1) N(N+1)^{3}(N+2)^{3}}-\frac{8 P_{25} S_{-2} S_{1}}{3(N-1) N^{2}(N+1)(N+2)^{2}} \\
& \left.+\frac{2\left(11 N^{4}+22 N^{3}-35 N^{2}-46 N-24\right) P_{19} S_{2}}{3(N-1)^{2} N^{3}(N+1)^{3}(N+2)^{3}}\right)+4 C_{A} T_{F}^{2}\left(\frac { 8 ( N ^ { 2 } + N + 2 ) } { 9 N ( N + 1 ) ( N + 2 ) } \left(S_{1}^{3}+8 S_{3}-12 S_{-2,1}\right.\right. \\
& \left.+9 S_{2} S_{1}+12 S_{-2} S_{1}+6 S_{-3}\right)-\frac{8\left(N^{3}+8 N^{2}+11 N+2\right) S_{1}^{2}}{3 N(N+1)^{2}(N+2)^{2}}-\frac{8 P_{19} S_{2}}{3(N-1) N^{2}(N+1)^{2}(N+2)^{2}} \\
& \left.+\frac{2 P_{26}}{27(N-1) N^{4}(N+1)^{4}(N+2)^{4}}+\frac{32\left(N^{2}-N-4\right) S_{-2}}{3(N+1)^{2}(N+2)^{2}}+\frac{4 P_{27} S_{1}}{27 N(N+1)^{3}(N+2)^{3}}\right)+2 C_{F}^{2} T_{F}( \\
& \frac{8\left(N^{2}+N+2\right)}{3 N(N+1)(N+2)}\left(4 S_{3} S_{1}-S_{1}^{4}-3 S_{2} S_{1}^{2}\right)+\frac{2\left(3 N^{4}+42 N^{3}+107 N^{2}+92 N+28\right) S_{1}^{3}}{3 N^{2}(N+1)^{2}(N+2)} \\
& +\frac{2 P_{28} S_{1}^{2}}{N^{3}(N+1)^{2}(N+2)}+\frac{2 P_{29} S_{1}}{N^{4}(N+1)^{4}(N+2)}+\frac{2\left(7 N^{4}+74 N^{3}+79 N^{2}-12 N-4\right) S_{2} S_{1}}{N^{2}(N+1)^{2}(N+2)} \\
& -\frac{8\left(N^{2}+N+2\right)\left(3 N^{2}+3 N+2\right) S_{3}}{3 N^{2}(N+1)^{2}(N+2)}-\frac{2\left(3 N^{2}+3 N+2\right)\left(N^{4}+17 N^{3}+17 N^{2}-5 N-2\right) S_{2}}{N^{3}(N+1)^{3}(N+2)} \\
& \left.-\frac{P_{30}}{N^{5}(N+1)^{5}(N+2)}\right)+4 C_{F} T_{F}^{2}\left(\frac{8\left(N^{2}+N+2\right)}{9 N(N+1)(N+2)}\left(4 S_{3}-S_{1}^{3}-3 S_{2} S_{1}\right)\right. \\
& +\frac{P_{31}}{3(N-1) N^{5}(N+1)^{5}(N+2)^{2}}+\frac{8\left(N^{4}-N^{3}-20 N^{2}-10 N-4\right) S_{1}}{3 N^{2}(N+1)^{2}(N+2)}+\frac{8\left(N^{4}+17 N^{3}+17 N^{2}-5 N-2\right) S_{2}}{3 N^{2}(N+1)^{2}(N+2)} \\
& \left.+\frac{8(3 N+2) S_{1}^{2}}{3 N^{2}(N+2)}\right)+2 C_{F} C_{A} T_{F}\left(\frac { 8 ( N ^ { 2 } + N + 2 ) } { 3 N ( N + 1 ) ( N + 2 ) } \left(2 S_{1}^{4}+12 S_{2} S_{1}^{2}+4 S_{3} S_{1}-12 S_{-2,1} S_{1}+6 S_{-3} S_{1}\right.\right. \\
& \left.+12 S_{-2} S_{1}^{2}\right)+\frac{4 P_{32} S_{1}^{3}}{9(N-1) N^{2}(N+1)^{2}(N+2)^{2}}-\frac{4 P_{33} S_{1}^{2}}{3(N-1) N^{3}(N+1)^{3}(N+2)^{3}} \\
& -\frac{4 P_{34} S_{1}}{3(N-1) N^{4}(N+1)^{4}(N+2)^{4}}-\frac{4 P_{35} S_{2} S_{1}}{3(N-1) N^{2}(N+1)^{2}(N+2)^{2}}-\frac{P_{36}}{3(N-1) N^{4}(N+1)^{5}(N+2)^{4}} \\
& -\frac{8 P_{37} S_{-2} S_{1}}{N^{2}(N+1)^{2}(N+2)^{2}}+\frac{4\left(N^{2}+N+2\right)\left(3 N^{2}+3 N+2\right)}{N^{2}(N+1)^{2}(N+2)}\left(2 S_{-2,1}-S_{-3}\right) \\
& -\frac{8\left(N^{2}-N-4\right)\left(3 N^{2}+3 N+2\right) S_{-2}}{N(N+1)^{3}(N+2)^{2}}-\frac{2 P_{38} S_{2}}{3(N-1) N^{3}(N+1)^{3}(N+2)^{2}}
\end{aligned}
$$




$$
\left.-\frac{8\left(N^{2}+N+2\right)\left(29 N^{4}+58 N^{3}-41 N^{2}-70 N-48\right) S_{3}}{9(N-1) N^{2}(N+1)^{2}(N+2)^{2}}\right) .
$$

Here, $S_{\vec{a}} \equiv S_{\vec{a}}(N), P_{k}$ denote some polynomials in $N$, cf. [17], and $\gamma_{i j}^{(2)}$ are the 3-loop anomalous dimensions. The expansion coefficients given above depend on harmonic sums up weight $\mathrm{w}=3$. Numerical studies show, that within the kinematic region of HERA the constant terms to (2.3) are as important as the logarithmic contributions. Further details will be given in [17].

\section{References}

[1] F. D. Aaron et al. [ H1 and ZEUS Collaborations ], JHEP 1001 (2010) 109. [arXiv:0911.0884 [hep-ex]].

[2] S. Alekhin, J. Blümlein and S. Moch, arXiv:1007.3657 [hep-ph];

S. Alekhin, J. Blümlein, S. Klein and S. Moch, Phys. Rev. D 81 (2010) 014032 [arXiv:0908.2766 [hep-ph]];

J. Blümlein, H. Böttcher and A. Guffanti, Nucl. Phys. B 774 (2007) 182 [arXiv:hep-ph/0607200]; Nucl. Phys. Proc. Suppl. 135 (2004) 152 [arXiv:hep-ph/0407089];

M. Glück, E. Reya and C. Schuck, Nucl. Phys. B 754 (2006) 178 [arXiv:hep-ph/0604116];

P. Jimenez-Delgado and E. Reya, Phys. Rev. D 79 (2009) 074023 [arXiv:0810.4274 [hep-ph]];

A. D. Martin, W. J. Stirling, R. S. Thorne and G. Watt, Eur. Phys. J. C 63 (2009) 189 [arXiv:0901.0002 [hep-ph]].

[3] M. Buza, Y. Matiounine, J. Smith, R. Migneron and W. L. van Neerven, Nucl. Phys. B 472 (1996) 611.

[4] J. A. M. Vermaseren, A. Vogt and S. Moch, Nucl. Phys. B 724 (2005) 3.

[5] I. Bierenbaum, J. Blümlein and S. Klein, Nucl. Phys. B 820 (2009) 417.

[6] M. Steinhauser, Comput. Phys. Commun. 134 (2001) 335 [arXiv:hep-ph/0009029].

[7] M. Buza, Y. Matiounine, J. Smith and W. L. van Neerven, Eur. Phys. J. C 1 (1998) 301, [hep-ph/9612398];

M. Buza, Y. Matiounine, J. Smith and W. L. van Neerven, Nucl. Phys. B485 (1997) 420, [hep-ph/9608342];

I. Bierenbaum, J. Blümlein and S. Klein, Nucl. Phys. B 780 (2007) 40; [hep-ph/0703285]; Phys. Lett. B 648 (2007) 195, [hep-ph/0702265]; Phys. Lett. B 672 (2009) 401, [hep-ph/0901.0669]; [arXiv:0706.2738 [hep-ph]] and in preparation;

I. Bierenbaum, J. Blümlein, S. Klein and C. Schneider, Nucl. Phys. B 803 (2008) 1, [hep-ph/0803.0273].

[8] J. Blümlein, A. De Freitas, W. L. van Neerven and S. Klein, Nucl. Phys. B 755 (2006) 272.

[9] J. Blümlein, S. Klein and B. Tödtli, Phys. Rev. D 80 (2009) 094010.

[10] J. Ablinger, J. Blümlein, S. Klein, C. Schneider and F. Wißbrock in preparation;

[11] J. Ablinger, I. Bierenbaum, J. Blümlein, A. Hasselhuhhn, S. Klein, C. Schneider and F. Wißbrock, arXiv:1007.0375 [hep-ph].

[12] C. Schneider, J. Symbolic Comput. 43 (2008) 611, [arXiv:0808.2543v1]; Ann. Comb. 9 (2005) 75; J. Differ. Equations Appl. 11 (2005) 799; Ann. Comb. (2009) to appear, [arXiv:0808.2596]; Proceedings of the Conference on Motives, Quantum Field Theory, and Pseudodifferential Operators, To appear in 
the Mathematics Clay Proceedings, 2010; Sém. Lothar. Combin. 56 (2007) 1, Article B56b, Habilitationsschrift JKU Linz (2007) and references therein;

J. Ablinger, J. Blümlein, S. Klein and C. Schneider, arXiv:1006.4797 [math-ph].

[13] J. Blümlein and S. Kurth, Phys. Rev. D 60 (1999) 014018 [arXiv:hep-ph/9810241];

J. A. M. Vermaseren, Int. J. Mod. Phys. A 14 (1999) 2037 [arXiv:hep-ph/9806280].

[14] S. A. Larin, T. van Ritbergen and J. A. M. Vermaseren, Nucl. Phys. B 427 (1994) 41;

S. A. Larin, P. Nogueira, T. van Ritbergen and J. A. M. Vermaseren, Nucl. Phys. B 492 (1997) 338; [hep-ph/9605317];

A. Retey and J. A. M. Vermaseren, Nucl. Phys. B 604 (2001) 281; [hep-ph/0007294];

J. Blümlein and J. A. M. Vermaseren, Phys. Lett. B 606 (2005) 130; [hep-ph/0411111];

[15] J. A. Gracey, Nucl. Phys. B662, 247 (2003) ; Nucl. Phys. B667, 242 (2003) ; JHEP 10, 040 (2006) ; Phys. Lett. B643, 374 (2006) ;

[16] J. A. Gracey, Phys. Lett. B 322 (1994) 141;

S. Moch, J. A. M. Vermaseren and A. Vogt, Nucl. Phys. B 688 (2004) 101; [hep-ph/0403192].

Nucl. Phys. B 691 (2004) 129; [hep-ph/0404111].

[17] I. Bierenbaum, J. Blümlein, and S. Klein, in preparation. 